\title{
Mixed Integer Programming with Decomposition to Solve a Workforce Scheduling and Routing Problem
}

\author{
Wasakorn Laesanklang, Dario Landa-Silva and J. Arturo Castillo-Salazar \\ School of Computer Science, ASAP Research Group, The University of Nottingham, Nottingham, U.K. \\ $\{$ psxwl3, dario.landasilva, psxjaca $\} @$ nottingham.ac.uk
}

\begin{abstract}
Keywords: Workforce scheduling, Routing problem, Home care scheduling, Mixed integer programming, Problem decomposition.

Abstract: $\quad$ We propose an approach based on mixed integer programming (MIP) with decomposition to solve a workforce scheduling and routing problem, in which a set of workers should be assigned to tasks that are distributed across different geographical locations. This problem arises from a number of home care planning scenarios in the UK, faced by our industrial partner. We present a mixed integer programming model that incorporates important real-world features of the problem such as defined geographical regions and flexibility in the workers' availability. Given the size of the real-world instances, we propose to decompose the problem based on geographical areas. We show that the quality of the overall solution is affected by the ordering in which the sub-problems are tackled. Hence, we investigate different ordering strategies to solve the sub-problems and show that such decomposition approach is a very promising technique to produce high-quality solutions in practical computational times using an exact optimization method.
\end{abstract}

\section{INTRODUCTION}

The workforce scheduling and routing problem (WSRP) is a difficult problem that arises in industries like home care, health care, patrol service, meter reading, etc. (Castillo-Salazar et al., 2014). One specific example of WSRP is home health care services where nurses or care workers should deliver care services at the patient's home. Solving the problem involves producing a job schedule and a route for each worker while satisfying the business requirements and considering workers qualifications and skills, task requirements, travelling distances, etc. It is usually expected that the solution gives the lowest operational cost.

Developing automated solution methods to solve WSRP scenarios is a current research challenge as reflected by recent published research (Mankowska et al., 2014; Liu et al., 2014; Hart et al., 2014). Solving an instance of WSRP often requires the expertise and knowledge of a human planner (Barrera et al., 2012). In this research, we are working with an industrial partner who provides scheduling services for businesses and other organisations facing this type of problems. The data sets considered here come from real-world scenarios. A particular feature is that "working areas' or 'regions' are pre-defined and this affects the difficulty of the problem being tackled.
Tackling WSRP with exact optimization methods to produce solutions in practice is still a research challenge. Since obtaining an optimal schedule is the ultimate goal, exact methods like mathematical programming are a suitable approach. However, proven optimality with exact methods has been shown mainly on problem instances of limited size (Borsani et al. 2006; Bredstrom and Ronnqvist, 2007). Problem instances faced in practice are larger and for solving them, heuristic methods are usually considered more efficient in terms of computation time (Akjiratikarl et al., 2007; Pillac et al., 2012). The motivation for our work is to develop a solution approach based on exact optimization to tackle real-world WSRP instances.

In this paper, we propose a decomposition approach that uses mixed integer programming to tackle WSRP instances of practical size. The proposed method splits the problem into sub-problems according to defined geographical areas. Our computational experiments show that the approach allows to explore the trade-off between computation efficiency and solution quality. Moreover, the insights from our experiments suggest that success of decomposition varies according to the problem instance, which provides us with ideas for future research.

The main contribution of this paper is to show that the ordering in which sub-problems in workforce 
scheduling and routing are tackled within a decomposition approach, has an effect on the computational efficiency and achieved solution quality. Following this, some ordering strategies are proposed to achieve solutions of good quality, typically $16.36 \%$ of relative gap on average, in practical computation time.

Section 2 reviews related works in the literature and establishes the motivation for the research in this paper. Section 3 gives the problem definition and formulation for the WSRP considered here. Section 4 describes the proposed MIP with decomposition approach and the experimental study, including description of test data instances. The final section summarises the paper and outlines some of the proposed future work.

\section{LITERATURE REVIEW}

Solving integer programming formulations for larger problem instances still has its limitations in terms of computation time. Mathematical programming has been used in the literature to tackle some WSRP. Examples include linear programming (Angelis, 1998), integer programming (Kergosien et al., 2009) and mixed integer programming (Bredstrom and Ronnqvist, 2007; Dohn et al., 2009; Borsani et al., 2006; Rasmussen et al., 2012). Solving real-world size problems often resources to heuristic or hybrid algorithms (Bertels and Torsten, 2006; Akjiratikarl et al., 2007; Eveborn et al., 2009). There are some improved exact methods, like branch and price (Bredstrom and Ronnqvist, 2007; Dohn et al., 2009; Rasmussen et al., 2012), that can deal with large instances. Branch and price requires problem reformulation which usually involves Dantzig-Wolfe decomposition to compute a tighter relaxation bound (Vanderbeck, 2000; Feillet, 2010). The algorithm also requires two steps to repeatedly solve the problem in order to improve the solution.

Decomposition techniques are another good alternative to apply exact optimization methods to large integer programming formulations. The basic idea here is to transform or split the problem into smaller subproblems. This technique has been applied in various problem domains. For example, Benders' decomposition was used to produce solutions for large instances of the aircraft routing and crew scheduling problem (Cordeau et al., 2001; Mercier et al., 2005). Benders' decomposition is suitable for problems with exclusive sub-problem sets or problems that shows some block structures linked by constraints (Benders, 1962). In another example (Perl and Daskin, 1985) split the warehouse location-routing problem into three smaller problems: the complete multi-depot vehicle-dispatch problem, the warehouse location-allocation problem and the multi-depot routing-allocation problem. These three smaller problems were solved in phases and each of them was formulated with mathematical programming and solved by an exact solver. For detailed reviews of decomposition approaches see (Ralphs and Galati, 2010; Vanderbeck and Wolsey, 2010).

Decomposition techniques have also been applied within heuristic approaches using some form of clustering. For example, (Reimann et al., 2004) tackled a large vehicle routing problem by decomposing it into sub-problems. Each sub-problem was a cluster of customers assigned to a vehicle which then became a travelling salesman problem. The sub-problem size is controlled by splitting a large sub-problem to shrink the corresponding cluster. Similar ideas were applied in a hybrid heuristic for generating multi-carrier transportation plans (Landa-Silva et al., 2011).

The purpose of this paper is to propose a decomposition approach that uses mixed integer programming (MIP) to tackle workforce scheduling and routing problem instances arising in real-world scenarios. For this, we also present an MIP formulation that incorporates features of the WSRP scenarios faced by our industrial partner. The proposed decomposition technique does not require some formulation structure like in Benders' decomposition neither uses a heuristic solver. Our approach harness the power of exact optimization solvers while decomposing the problem instances in a way that is meaningful to practice.

\section{PROBLEM DESCRIPTION AND FORMULATION}

The goal in WSRP is to assign each worker to undertake a set of tasks across a set of geographical locations. A path is the series of tasks to be performed by a worker within the planning period. A good quality solution consists of a set of shortest paths representing the task assignments for each worker at the lowest cost. The solution should also respect other conditions such as task pre-requisites, required travelling time between locations, defined appointment times, workers' skills, workers availability, restricted working regions, working time limits, etc.

Consider a graph $G=(V, E)$ where $V=T \cup D \cup$ $D^{\prime}$ represents the union of a set of tasks (each task as a location) $T$, a set of start locations $D$ and a set of end locations $D^{\prime}$ while $E$ represents a set of links between two locations (e.g. between two task locations or between the worker's home and a task location). The set of workers is denoted by $C$. Binary decision variable $x_{i, j}^{c}=1$ if worker $c \in C$ is assigned to a task $j \in T$ after finishing task $i \in T, x_{i, j}^{c}=0$ otherwise. 
Note that elements of $T$ are referred here as tasks but also each task has an associated location.

In practical scenarios like the ones considered here, the available workforce and its skills are often not sufficient to cover all the tasks that need performing. This can be addressed by introducing a dummy worker (through a binary decision variable $y_{j}$ ) that takes any excess work that cannot be assigned to the real workforce (Bredstrom and Ronnqvist, 2008; Rasmussen et al., 2012). Then, the assignment of tasks is represented by (1).

$$
\sum_{c \in C} \sum_{i \in D \cup T} x_{i, j}^{c}+y_{j}=1 \quad, \forall j \in T
$$

The sequence of tasks that a worker performs is represented as a path for visiting task locations, hence the number of workers arriving at one location must be equal to the number of workers leaving that task location so that either workers are assigned to the next task or go home. Then, the path constraint is represented by (2).

$$
\sum_{i \in D \cup T} x_{i, j}^{c}=\sum_{k \in D^{\prime} \cup T} x_{j, k}^{c} \quad, \forall j \in T, \forall c \in C
$$

Workers must start and end their paths from their specific location (e.g. their home or a central office) as given by (3) and (4). Since $D$ and $D^{\prime}$ are sets of start and end locations respectively, these two constraints indicate the start and end locations for each worker. Also, workers leave their start location and enter their end location at most once (although the start and end locations can be different) as expressed by (5) and (6) respectively. Note that a worker does not leave his start location if he is not assigned to work. This is different from the common case in the literature where all workers leave their start location. In our problem instances, the specific start and end locations are provided for every worker.

$$
\begin{aligned}
& \sum_{j \in D^{\prime} \cup T} x_{k, j}^{c} \geq \sum_{j \in D^{\prime} \cup T} x_{i, j}^{c} \\
& \qquad, \forall c \in C, \forall i \in T, \exists k \in D
\end{aligned}
$$

$$
\begin{gathered}
\sum_{i \in D \cup T} x_{i, k}^{c} \geq \sum_{i \in D \cup T} x_{i, j}^{c} \\
\sum_{j \in D^{\prime} \cup T} x_{i, j}^{c} \leq 1 \quad, \forall c \in C, \forall j \in T, \exists k \in D^{\prime} \\
\sum_{i \in D \cup T} x_{i, j}^{c} \leq 1 \quad, \forall j \in D^{\prime}, \forall c \in C
\end{gathered}
$$

Let $S$ be the set of skills and $s \in S$ a particular skill. For worker $c$ the qualification level on skill $s$ is $q_{s}^{c}$ and for task $j$ the requirement of skill $s$ is $r_{s, j}$. Hence, worker $c$ can be assigned to task $j$ only if the worker has the required qualifications level for skill $s$, that is, $q_{s}^{c} \geq r_{s, j}$. Then, in our model the multi-skill qualification requirements are represented by (7).

$$
\begin{aligned}
& x_{i, j}^{c} r_{s, j} \leq q_{s}^{c} \\
& \quad, \forall c \in C, \forall i \in D \cup T, \forall j \in T, \forall s \in S
\end{aligned}
$$

Also, travel time between task locations must be feasible. Decision variable $a_{i}^{c}$ takes a positive fractional value that gives the worker arrival time to the location of task $i$. Note that the maximum arrival time value is 1440 which is equivalent to the $24^{\text {th }}$ hour of the day. Let $a_{i}^{c}, a_{j}^{c}$ be the arrival times of worker $c$ to the locations of task $i$ and task $j$ respectively. Let $t_{i, j}$ be the travelling time between the locations of tasks $i$ and $j$. Let $\delta_{i}$ be the duration of task $i$. Then, if worker $c$ is assigned to perform task $j$ after completing task $i$, inequality (8) ( $M$ is a large constant number) expresses the arrival on time requirement.

$$
\begin{aligned}
a_{j}^{c}+M\left(1-x_{i, j}^{c}\right) \geq a_{i}^{c}+x_{i, j}^{c} t_{i, j}+\delta_{i} \\
\quad, \forall c \in C, \forall i \in D \cup T, \forall j \in D^{\prime} \cup T
\end{aligned}
$$

An arrival time window is also defined for task $i$ and the worker should not arrive earlier than $w_{i}^{L}$ or later than $w_{i}^{U}$, as expressed by (9).

$$
w_{i}^{L} \leq a_{i}^{c} \leq w_{i}^{U} \quad, \forall i \in T, \forall c \in C
$$

An important feature of our WSRP scenarios is that working regulations and availability can be specific for each worker. In the problems considered here, this refers to long breaks between shifts (short breaks within the working shift are not considered), daysoff, working shift duration, maximum working hours, and specific worker preferences (e.g. late morning, afternoon only, whole day, overnight). We adopt a flexible availability constraint from an optimization of daily scheduling for home health care services (Trautsamwieser and Hirsch, 2011). Any task assignment at time $a_{j}^{c}$ including the task duration $\delta_{j}$ should lie in between the shift starting time $\alpha_{L}^{c}$ and the shift ending time $\alpha_{U}^{c}$. The availability parameters $\alpha_{L}^{c}$ and $\alpha_{U}^{c}$ are real numbers defined for each worker $c$. A task assigned outside the shift is charged as additional expense, hence binary decision variable $\omega_{j}=1$ if this is the case and $\omega_{j}=0$ otherwise. Then, individual availability constraints are denoted by (10) and (11) while the working hours limit $\left(h^{c}\right)$ constraint is denoted by (12).

$$
\begin{aligned}
\alpha_{L}^{c}-a_{j}^{c} \leq M(1- & \left.x_{i, j}^{c}+\omega_{j}\right) \\
& \forall c \in C, \forall i \in D \cup T, \forall j \in T
\end{aligned}
$$




$$
\begin{gathered}
a_{j}^{c}+\delta_{j}-\alpha_{U}^{c} \leq M\left(1-x_{i, j}^{c}+\omega_{j}\right) \\
\forall c \in C, \forall i \in D \cup T, \forall j \in T \\
\sum_{i \in D \cup T} \sum_{j \in T} x_{i, j}^{c} \delta_{j} \leq h^{c} \quad, \forall c \in C
\end{gathered}
$$

Another important feature of our WSRP scenarios is that workers have preferred geographical areas for working but the decision maker can still request workers to work outside those preferred regions. We formulate this in (13) where binary parameter $\gamma_{j}^{c}=1$ if worker $c$ is willing and able to work at the location of task $j, \gamma_{j}^{c}=0$ otherwise, and binary decision variable $\psi_{j}=1$ if worker $c$ is forced to work outside their defined regions.

$$
\sum_{i \in D \cup T} x_{i, j}^{c}-\psi_{j} \leq \gamma_{j}^{c} \quad, \forall c \in C, \forall j \in T
$$

Most of the above constraint formulations exist in literature but not all. Common constraints (see also (Castillo-Salazar et al., 2014)) such as path constraint (2), skill and qualification (7) and time windows (9) form the basic structure of the scheduling and routing problem (Dantzig and Ramser, 1959; Bredstrom and Ronnqvist, 2008; Dohn et al., 2009; Castro-Gutierrez et al., 2011; Rasmussen et al., 2012). Tailor cut constraints adopted from literature are the availability constraints $(10,11)$ while the constraints that required further adaptation to our problem features are the working region (13) (implemented as soft constraint) and startend paths (3-6).

Table 1 summarises the constraints in our MIP model. Given our real-world data sets, some are implemented as soft constraints. For example, workers can be forced to work outside their predefined regions and availability. Also, tasks can be left unassigned (assigned to the dummy worker). These features are quite important to maintain the practical applicability of our model and solution approach.

The objective function (14) involves three costs: monetary cost, preferences penalty cost and soft constraints penalty cost.

$$
\begin{aligned}
& \operatorname{Min} \sum_{c \in C} \sum_{i \in D \cup T} \sum_{j \in D^{\prime} \cup T}\left(d_{i, j}+p_{j}^{c}\right) x_{i, j}^{c} \\
& +\sum_{c \in C} \sum_{i \in D \cup T} \sum_{j \in D^{\prime} \cup T} \rho_{j}^{c} x_{i, j}^{c} \\
& \quad+\sum_{j \in T} M_{1} y_{j}+M_{2}\left(\omega_{j}+\psi_{j}\right)
\end{aligned}
$$

The first term in (14) is the monetary cost and includes the travelling cost $d_{i, j}$ of going from location of task $i$ to the location of task $j$, and the payment $p_{j}^{c}$ for
Table 1: Summary of problem requirements and type of constraints.

\begin{tabular}{lcc}
\hline & Hard & Soft \\
\hline Job assignment (Cons. (1)) & & $*$ \\
Path constraint (Cons. (2)) & $*$ & \\
Start-end paths (Cons. (3) - (6)) & $*$ & \\
Skill and qualification (Cons. (7)) & $*$ & \\
Travel time feasibility (Cons. (8)) & $*$ & \\
Time windows (Cons. (9)) & $*$ & \\
Worker availability (Cons. (10),(11)) & $\dagger$ & $*$ \\
Working hours limit (Cons. (12)) & $*$ & \\
Working regions (Cons. (13)) & & $*$ \\
\hline
\end{tabular}

$\dagger$ Hard constraints (15) and (16) are described in Section 4.2 and only apply to the decomposition model.

worker $c$ to perform task $j$. The second term in (14) is the preference penalty cost denoted by $\rho_{j}^{c} \geq 0$ and is a summation of penalties for not meeting worker-client preferences, required skill preferences and working region preferences. This penalty value can go from 0 to 75 and $\rho_{j}^{c}=0$ when all preferences are met, while this penalty value grows higher as the preference level of assigning worker $c$ to task $j$ decreases. The third term in (14) is the soft constraints penalty cost due to the violation of the three soft constraints in the model. The job assignment constraint has the highest priority $\left(M_{1}=2000\right)$, so a violation of this constraint type costs more than a violation of the the other two constraints. The worker availability and working regions constraints have the same priority $\left(M_{2}=500\right)$. Note that the working regions constraint is involved in two costs. If the worker is assigned a task in a non-preferred region then this is a constraint penalty cost. If the worker is assigned to one of the preferred regions this is quantified as a preference penalty cost according to the degree in which the region is preferred by the worker (several working regions with different preference levels).

The above MIP model corresponds to the integrated scheduling and routing problem. Solving this model with an exact optimization method is not practical considering our real-world problem instances. Hence, we apply a decomposition technique.

\section{DECOMPOSITION APPROACH AND STUDY}

In order to reduce the overall computational time of solving real-world instances of the integrated workforce scheduling and routing problem, we propose a decomposition method in this section. First, we describe the features of our problem instances as this will help to explain the proposed decomposition approach. 
Later, the method is described and experimental results are presented.

\subsection{Test Instances}

For the present work, we prepared some test instances using real-world data corresponding to home care scenarios in the UK, provided by our industrial partner. A problem instance $P$ has a set of locations $V$. Recall from Section 3 that $V=D \cup T \cup D^{\prime}$. Also, some of the tasks $\left\{j_{1}, j_{2}, \ldots, j_{n}\right\}$ in $T$ share the same geographical location $\kappa \in K$, where $K$ is a set of geographical locations. A group of locations are assembled as a geographical area or working region $a \in A$. Note that $a \subseteq K$ and $A$ is a partition. Also, an individual worker $c$ may work on one or several geographical areas. As noted above, a key aspect of our scenarios is that several tasks might be required at one particular location. Each individual task may have different required skills, worker preferences and worker cost.

We took four real-world scenarios and prepared a data set from each. Although the instances in each data set come from the same scenario, each instance is formed from a different planning time giving a variation in the available human resources and task requirements. In our data, the start and end locations of a worker are the same $\left(d=d^{\prime}\right)$. Table 2 shows the main features of the test instances: the number of available workers $|C|$, the number of task locations $|K|$, the numbers of tasks $|T|$ and the number of predefined geographical areas $|A|$. In terms of size, instances WSRP-A-(01-07) and WSRP-B-(01-07) are considered small with around 19-34 workers and 13-69 tasks. The optimal solution for each of these instances can be found in less than 5 minutes. Instances WSRP-D-(0107) and WSRP-F-(01-07) are considered large with more than 100 workers and 400 tasks. These large instances cannot yet be solved to optimality in practical computation time. In our experimental study, we use the small instances to validate the proposed decomposition approach as we can compare to the optimal solutions. Moreover, the experimental results show the suitability of the decomposition approach in tackling the large instances using an exact optimization solver.

\subsection{Geographical Decomposition with Conflict Avoidance}

In this paper, the workforce scheduling and routing problem is decomposed into working regions as this is a key feature of the scenarios provided by our industrial partner. Since we decompose the problem into sub-problems to deal with the larger size more efficiently, by solving the sub-problems one at a time in
Table 2: The test data sets.

\begin{tabular}{llllr}
\hline Instance & $|C|$ & $|K|$ & $|T|$ & $|A|$ \\
\hline WSRP-A-01 & 23 & 25 & 31 & 6 \\
WSRP-A-02 & 22 & 24 & 31 & 4 \\
WSRP-A-03 & 22 & 28 & 38 & 5 \\
WSRP-A-04 & 19 & 22 & 28 & 3 \\
WSRP-A-05 & 19 & 9 & 13 & 3 \\
WSRP-A-06 & 21 & 22 & 28 & 7 \\
WSRP-A-07 & 21 & 9 & 13 & 3 \\
\hline WSRP-B-01 & 25 & 27 & 36 & 6 \\
WSRP-B-02 & 25 & 11 & 12 & 4 \\
WSRP-B-03 & 34 & 43 & 69 & 6 \\
WSRP-B-04 & 34 & 14 & 30 & 4 \\
WSRP-B-05 & 32 & 38 & 61 & 8 \\
WSRP-B-06 & 32 & 38 & 57 & 7 \\
WSRP-B-07 & 32 & 38 & 61 & 7 \\
\hline WSRP-D-01 & 164 & 233 & 483 & 13 \\
WSRP-D-02 & 166 & 215 & 454 & 12 \\
WSRP-D-03 & 174 & 279 & 585 & 15 \\
WSRP-D-04 & 174 & 237 & 520 & 15 \\
WSRP-D-05 & 173 & 259 & 538 & 15 \\
WSRP-D-06 & 174 & 291 & 610 & 15 \\
WSRP-D-07 & 173 & 293 & 611 & 15 \\
\hline WSRP-F-01 & 805 & 477 & 1211 & 45 \\
WSRP-F-02 & 769 & 496 & 1243 & 46 \\
WSRP-F-03 & 898 & 582 & 1479 & 54 \\
WSRP-F-04 & 789 & 513 & 1448 & 47 \\
WSRP-F-05 & 883 & 626 & 1599 & 59 \\
WSRP-F-06 & 783 & 565 & 1582 & 44 \\
WSRP-F-07 & 1011 & 711 & 1726 & 64 \\
\hline$|C|=$ number of workers. & & \\
$|K|=$ number of task locations. & & \\
$|T|=$ number of required tasks. & & \\
$|A|=$ number of working regions. & & \\
& & & &
\end{tabular}

a given sequence, we can no longer guarantee overall optimality.

Basically, the decomposition method generates a sub-problem for each working region and solves each sub-problem in sequence. Worker assignment conflicts (i.e. a worker being assigned to different task locations at the same time) are avoided because each sub-problem is solved using only the reduced available workforce after solving the previous sub-problem.

Algorithm 1 presents the proposed geographical decomposition with conflict avoidance approach (GDCA). A problem instance $P$ is split into several subproblems $P_{i}$ (step 2). A sub-problem $P_{i}$ corresponds to a geographical area or working region $a \in A$. Then, the sub-problems are solved in a given sequence (steps 3 6) and different solving sequences can lead to different solution quality. This is because the first sub-problem has access to the most workforce resources but subsequent sub-problems will have access to limited available workforce. Since worker assignment conflicts are avoided, this means that the hard constraints ex- 
pressed by equations (15) and (16) are enforced in this algorithm.

$$
\begin{aligned}
& a_{j}^{c}+\delta_{j}-\beta_{L}^{c} \leq M\left(2-x_{i, j}^{c}-\zeta^{c}\right) \\
& \forall c \in C, \forall i \in D \cup T, \forall j \in T \cup D^{\prime} \\
& \beta_{U}^{c}-a_{j}^{c} \leq M\left(1-x_{i, j}^{c}+\zeta^{c}\right) \\
& \forall c \in C, \forall i \in D \cup T, \forall j \in T \cup D^{\prime}
\end{aligned}
$$

Here, $\beta_{L}^{c}$ denotes the start of unavailable time and $\beta_{U}^{c}$ denotes the end of unavailable time for worker $c$. Since the original model generates a continuous path for a worker, a path created under hard availability conditions is allocated either before or after the unavailability period. That is, a path which overlaps with the unavailability period defined by $\beta_{L}^{c}$ and $\beta_{U}^{c}$ is not allowed. The control variable $\zeta^{c}$ is applied for selecting only one side of the availability period. When $\zeta^{c}=1$ the time interval before $\beta_{L}^{c}$ is selected and if the $\zeta^{c}=0$ the time interval after $\beta_{U}^{c}$ is selected.

In our data, we know that the start location $d$ and end location $d^{\prime}$ for a worker are the same. Therefore, we designed a sub-problem solutions combination process based on this assumption. During the Combine sub-problem solutions process (step 7), sub-problem solutions are combined together by connecting the worker's paths from each sub-problem to get a long single path. After this process, a worker leaves his start location and arrives to his end location only once. Suppose that $\Phi_{1}=\left\{\left(x_{d, t_{1}}^{c}, a_{1}^{c}\right),\left(x_{t_{1}, d^{\prime}}^{c}, a_{d_{1}}^{c}\right)\right\}$ is a solution to sub-problem $P_{1}$ representing the assignment of worker $c$ from start location $d$ to work on task $t_{1}$ and returning to end location $d^{\prime}$ and $\Phi_{2}=$ $\left\{\left(x_{d, t_{2}}^{c}, a_{2}^{c}\right),\left(x_{t_{2}, d^{\prime}}^{c}, a_{d_{2}}^{c}\right)\right\}$ is a solution to sub-problem $P_{2}$ representing the assignment of the same worker $c$ from starting location $d$ to work on task $t_{2}$ and returning to ending location $d^{\prime}$. Assume without loss

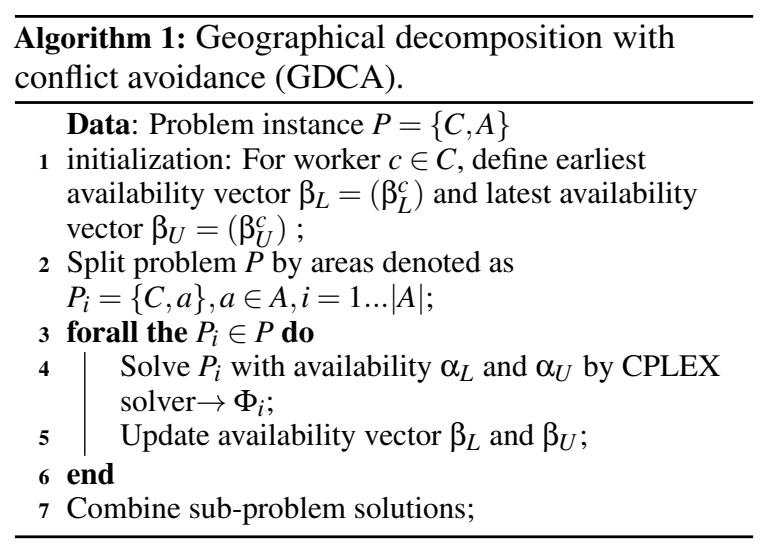

of generality that $a_{1}^{c}<a_{d_{1}}^{c}<a_{2}^{c}<a_{d_{2}}^{c}$. The combining process redirects the arriving assignment to end location $d^{\prime}$ to task $t_{2}$ which gives a global solution as $\Phi=\left\{\left(x_{d, t_{1}}^{c}, a_{1}^{c}\right),\left(x_{t_{1}, t_{2}}^{c}, a_{2}^{c}\right),\left(x_{t_{2}, d^{\prime}}^{c}, a_{d_{2}}^{c}\right)\right\}$. It is possible than in other scenarios of the WSRP, the start location and end location for a worker are different, we leave this for future work as it is not a feature of the scenarios tackled at present.

\subsection{Experiments and Results}

The flow of our experimental study is depicted in Figure 1 . The figure outlines the three parts of the experimental design. First, on the left-hand side of the figure, the permutation study refers to solving the sub-problems in different order given by all the different permutations of the geographical areas. However, trying all permutations is practical only in small problems. Therefore, finding an effective ordering pattern is the second part of experiment, observation step in the figure. This second part solved each sub-problem using all available workforce, i.e. ignoring if some workers were assigned in previous sub-problems. The third part analysed the results from the observation step in order to define some strategies to tackle the sub-problems. Based on this strategies study, some solving strategies were envisaged. Listed in the figure are these ordering strategies: Asc-task, Desc-task, Ascw\&u, etc. More details about these ordering strategies are provided when decribing the Observation step below. Finally, the solutions produced with the different ordering strategies are compared to the solutions produced by the permutation study to evaluate the performance of these ordering strategies.

Permutation Study. Since the number of permutations grows exponentially with the number of geographical areas, we performed the permutation study using only the instances with $|A|=3$ and $|A|=4$ geographical areas. Figure 2 shows the relative gap obtained for the small instances that have 3 regions. Each sub-figure shows the results for one instance when solved using the different permutation orders of the 3 regions. Each bar shows the relative gap between the solution by the decomposition method and the overall optimal solution. The figure shows that the quality of the obtained solutions for the different permutations fluctuates considerably. Closer inspection reveals that in these instances the geographical areas are very close to each other and sometimes there is an overlap between them. The result also reveals that some permutations clearly give better results. For example, permutation "1-2-3" for instance WSRP-A-04, permutations "1-2-3" and "2-1-3" for instance WSRP-A-05 and permutations " $1-2-3$ " and "1-3-2" for instance 


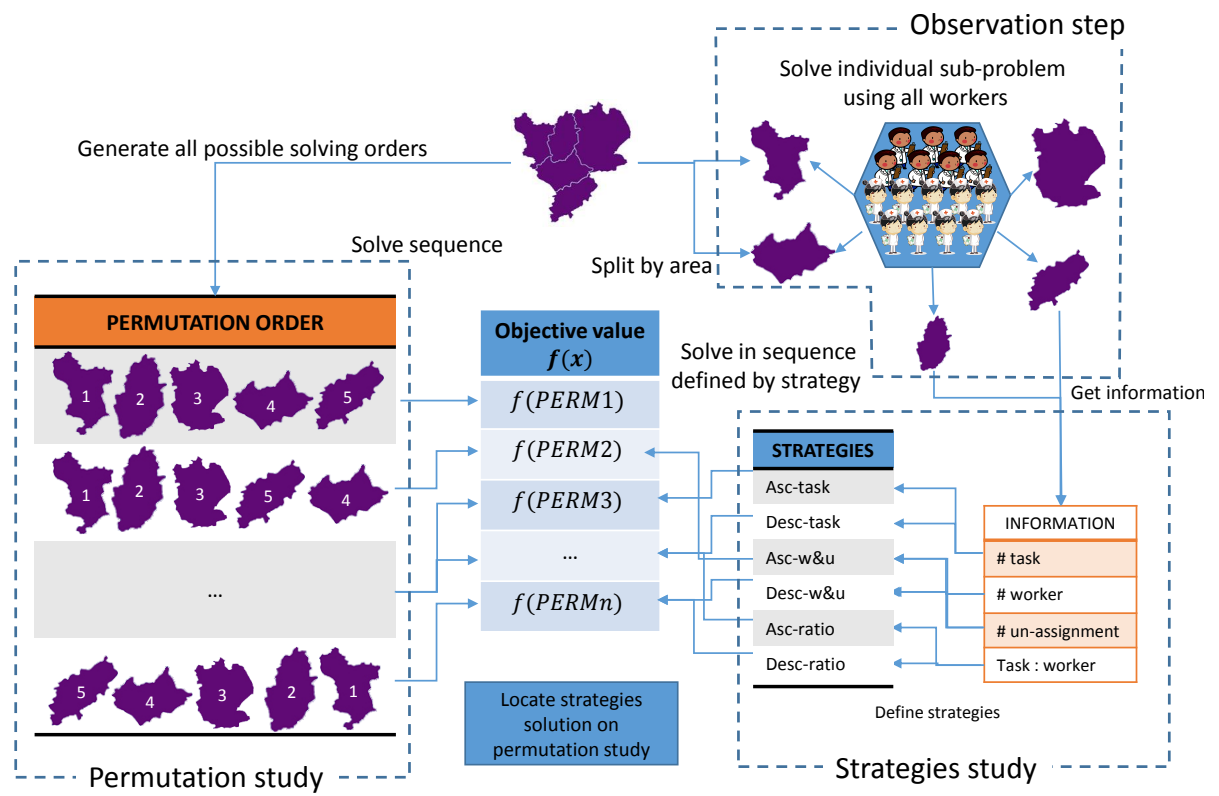

Figure 1: Outline of the experimental study in three parts: permutation study, observation step and strategies study.

WSRP-A-04

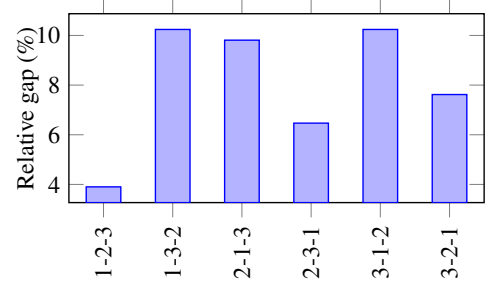

WSRP-A-05

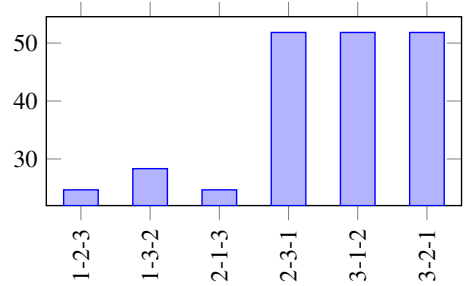

WSRP-A-07

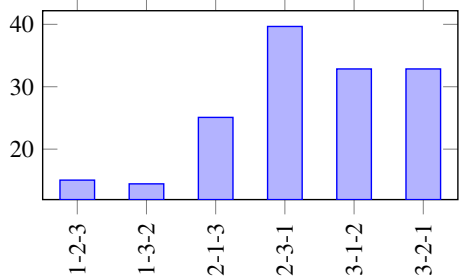

Figure 2: Relative gap obtained from solving the 3 instances (WSRP-A-04, WSRP-A-05 and WSRP-A-07) with $|A|=3$ using the different permutation orders. Each graph shows results for one instance. The bars represent the relative gap between the solution obtained with the decomposition method and the overall optimal solution.

\section{WSRP-A-07.}

Figure 3 shows the relative gap obtained for the small instances that have 4 regions. Each sub-figure shows the result for one instance when solved using the permutation orders of the 4 regions. Each bar shows the relative gap between the solution by the decomposition method and the overall optimal solution. The figure reveals an interesting result from instance WSRP-B-02. The optimal solution value is obtained for every permutation. Closer inspection reveals that the decomposition method works very well on this instance because its geographical areas are well separated from each other. Therefore, the sub-problem solutions are part of the complete overall solution and not many worker assignment conflicts arise when solving the sub-problems. For the other instances, WSRPA-02 and WSRP-B-04, the quality of the obtained solutions fluctuates in the same way as in Figure 2. Results in Figure 3 indicate that some solutions obtained with the decomposition approach using some permu- tations have a considerable gap in quality compared to the overall optimal solution. The figure also shows that some permutations clearly give better results than others. For example, permutation "1-2-3-4", "2-1-3-4" and "2-3-1-4" for instance WSRP-A-02 and permutation "4-3-2-1" for instance WSRP-B-04.

The conclusion from this permutation study is that the order in which the sub-problems are solved matters differently according to the problem instance. More importantly, the results confirm our assumption that some particular permutation could produce a very good result in the decomposition approach. Hence, the next part of the study is to find a good solving order.

Observation Step. Here we solve each of the subproblems using all available workers and collect the following values from the obtained solutions: number of tasks in the sub-problem (\# task), minimum number of workers required in the solution (\# min worker), number of unassigned tasks in the solution (\# unas- 


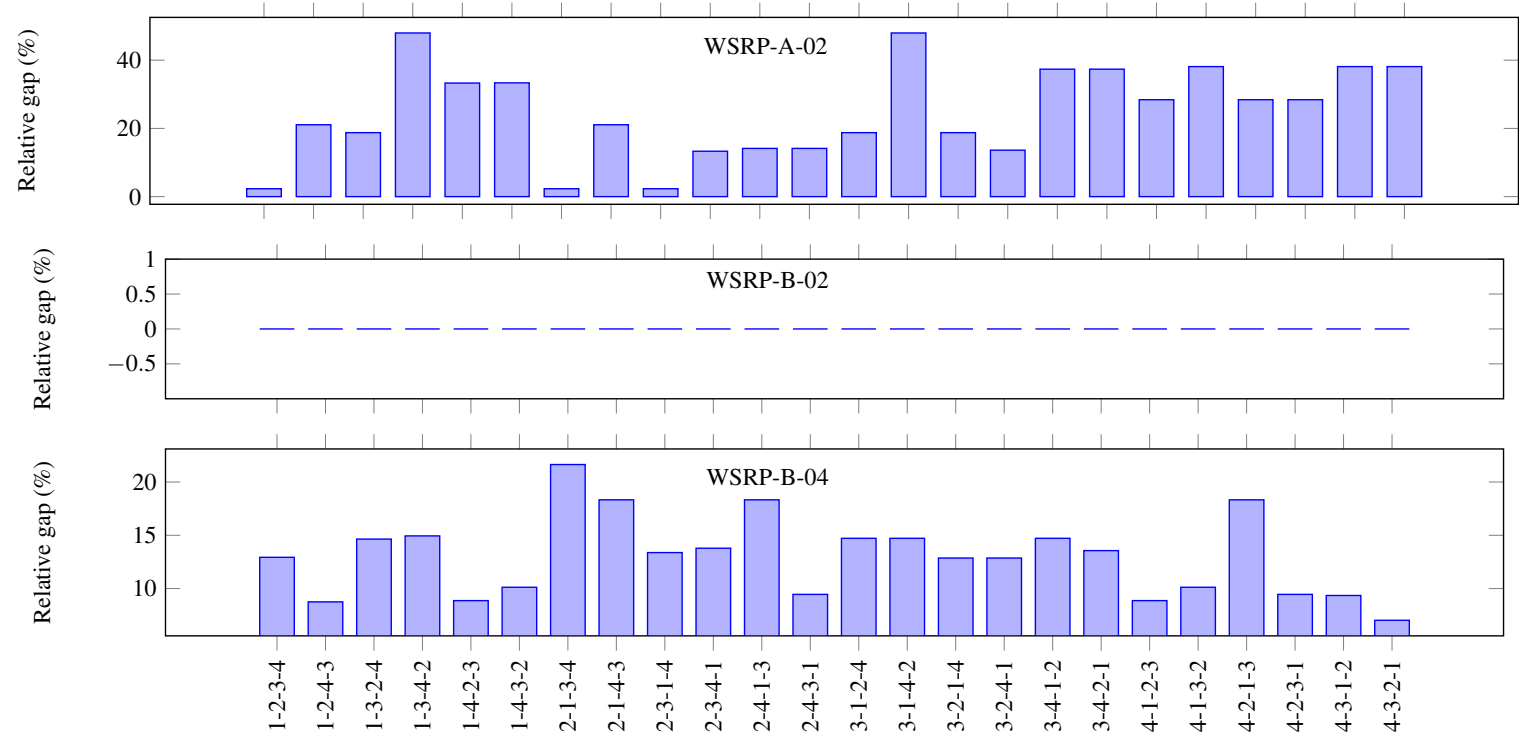

Figure 3: Relative gap obtained from solving the 3 instances (WSRP-A-02, WSRP-B-02 and WSRP-B-04) with $|A|=4$ using the different permutation orders. Each graph shows results for one instance. The bars represent the relative gap between the solution obtained with the decomposition method and the overall optimal solution.

signed task) and the ratio of tasks to worker in the solution (task/worker ratio). Then, we defined six ordering strategies as follows. Increasing number of tasks in the sub-problem (Asc-task); decreasing number of tasks in the sub-problem (Desc-task); increasing sum of minimum workers required and unassigned tasks (Asc$\mathrm{w} \& u$ ); decreasing sum of minimum workers required and unassigned tasks (Desc-w\&u); increasing ratio of tasks to worker (Asc-ratio) and decreasing ratio of tasks to worker (Desc-ratio).

Strategies Study. The GDCA approach is again executed using the 6 ordering strategies listed above to tackle the sub-problems in each problem instance. The results are presented in Figure 4 which shows the relative gap for the 14 small instances in the WSRP-A and WSRP-B groups. Note that each bar represents the relative gap obtained with each strategy.

From Figure 4, the decomposition technique with ordering strategies gives solutions with relative gaps below $50 \%$. On average, the decomposition technique produces relative gap at $16.36 \%$. Moreover, we can see that some of the ordering strategies are more likely to produce better solutions than others. The best performing ordering strategy is Asc-w\&u that gives 9 best solutions considering all 14 small instances. The average gap for the ordering strategies Asc-task, Desctask, Asc-w\&u, Desc-w\&u, Asc-ratio and Desc-ratio are $14.09 \%, 19.94 \%, 11.19 \%, 19.66 \%, 15.01 \%$ and $18.28 \%$ respectively. Table 3 shows a comparison of relative gap between the best permutation order (see Permutation study) and the best ordering strategy. It
Table 3: Relative gap (\%) of best permutation VS. best strategy.

\begin{tabular}{lcc}
\hline Instance & Best permutation & Best strategy \\
\hline WSRP-A-04 & 3.9 & 6.4 \\
WSRP-A-05 & 24.67 & 24.67 \\
WSRP-A-07 & 14.43 & 15.03 \\
WSRP-A-02 & 2.31 & 2.32 \\
WSRP-B-02 & 0 & 0 \\
WSRP-B-04 & 7.02 & 8.86 \\
\hline
\end{tabular}

is clearly shown that solutions from the best ordering strategy are not much different from the best permutation solution (maximum of $2.5 \%$ different). In addition, two out of six solutions (instance WSRP-A-05 and WSRP-B-02) of the best ordering strategy match the solution from the best permutation. This shows that the ordering strategies are able to work well in other problem instances.

The decomposition method is also able to find solutions for the large instances whilst solving those problems as a whole is not practical in terms of computation time. The results from using the decomposition technique with the 6 ordering strategies on the large instances are presented in Table 4. The table shows the objective values of the obtained solutions as relative gaps cannot be computed because the optimal solutions are not known. The values in bold are the lowest cost (best objective value) obtained among the six strategies. The table shows that as a whole, the Desc-ratio and Asc-w\&u give four and three best 


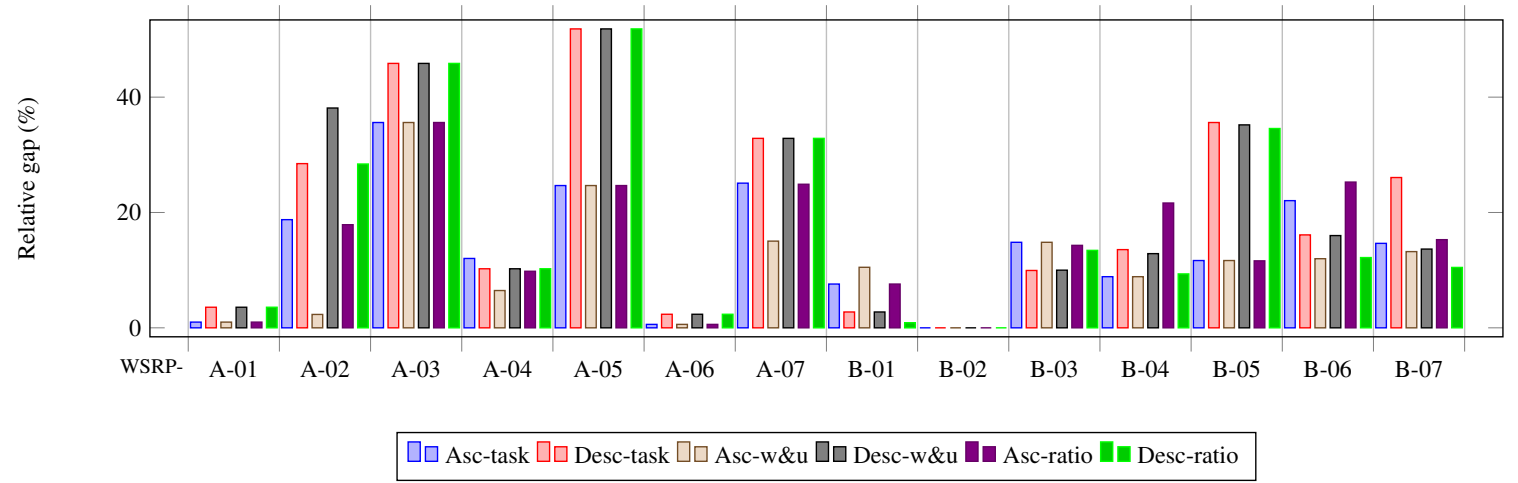

Figure 4: Relative gap obtained from solving the 14 small instances using the 6 ordering strategies. Each bar for an instance represents the relative gap between a solution by the decomposition method using an ordering strategy and the overall optimal solution.

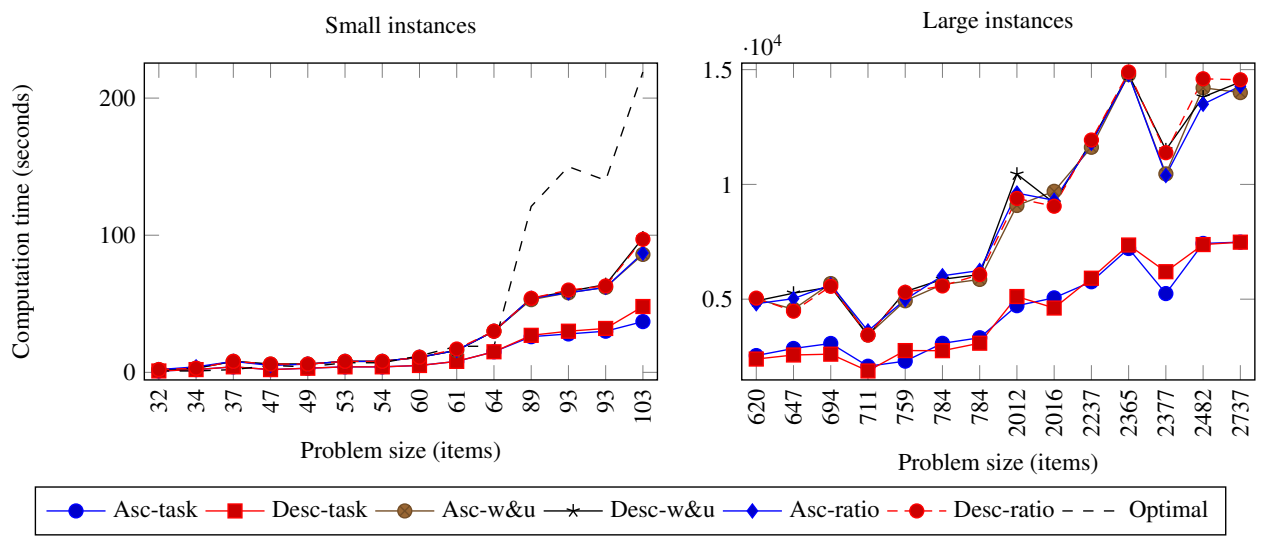

Figure 5: Computation time (seconds) used in solving small and large instances. Each sub-figure corresponds to a problem size category (small and large). The problem size (items) is the summation of \#workers and \#tasks. Each graph presents the computation time used by the decomposition method with the different ordering strategies (line with markers) and the time used for producing the overall optimal solution (dashed line) when possible.

solutions respectively while the others give two best solutions. On average, the Desc-task strategy gives the lowest cost solution, around $5.7 \%$ less than the highest average cost strategy (Asc-ratio).

Figure 5 shows, according to the problem size, the computation times used by the decomposition approach using the different ordering strategies and the time used to find the overall optimal solution. Each sub-figure presents the problem instances classified by their size (number of items is $|T|+|C|$ ). Each line represents the time used by the ordering strategy in solving the group of 14 problem instances. As noted before, the time to find the optimal solution represented by --- is available only for the small instances. For the smaller instances with less than 89 items, the computation time used by the decomposition method is not much different from the time used to find the optimal solution. The computation time used to find the optimal solution grows significantly for instances with 89 items and above. For the large instances, it is shown that the computation time used by the decomposition method goes above 4 hours $(14,895$ seconds). Also, for the large instances the computation time used by the ordering strategies Asc-task and Desc-task is significantly less than for the other ordering strategies. This is because these ordering strategies do not require an additional process to retrieve information about the problem. Hence, considering both solution quality and computation time, it can be concluded that Asc-task and Desc-task (best known on average) should be selected for large instances because they produce solutions which are not much different from the other strategies but requiring significantly less computational time ( $48.3 \%$ less on average).

\section{CONCLUSION AND FUTURE WORK}

A tailored mixed integer programming model for realworld instances of a workforce scheduling and routing 
Table 4: Objective value obtained from solving large instances using six ordering strategies.

\begin{tabular}{ccccccc}
\hline Instance & Asc-task & Desc-task & Asc-w\&u & Desc-w\&u & Asc-ratio & Desc-ratio \\
\hline WSRP-D-01 & $118,647.95$ & $109,634.79$ & $120,538.27$ & $\mathbf{1 0 7 , 2 6 4 . 9 3}$ & $112,695.33$ & $109,693.81$ \\
WSRP-D-02 & $119,505.44$ & $120,707.43$ & $117,169.05$ & $119,317.34$ & $\mathbf{1 1 3 , 3 6 7 . 9 2}$ & $115,790.07$ \\
WSRP-D-03 & $95,302.20$ & $93,097.74$ & $95,302.20$ & $92,349.70$ & $95,302.20$ & $\mathbf{8 9 , 4 6 8 . 6 3}$ \\
WSRP-D-04 & $103,685.14$ & $\mathbf{1 0 1 , 8 6 1 . 7 4}$ & $250,740.31$ & $135,675.84$ & $105,268.17$ & $143,621.85$ \\
WSRP-D-05 & $86,581.13$ & $84,505.24$ & $\mathbf{8 4 , 3 6 6 . 4 8}$ & $87,588.51$ & $86,581.13$ & $84,505.24$ \\
WSRP-D-06 & $76,681.66$ & $77,279.43$ & $74,438.87$ & $80,083.86$ & $76,737.43$ & $\mathbf{7 3 , 2 0 2 . 0 4}$ \\
WSRP-D-07 & $\mathbf{7 1 , 0 2 9 . 1 1}$ & $77,381.16$ & $71,485.90$ & $117,757.75$ & $\mathbf{7 1 , 0 2 9 . 1 1}$ & $73,055.89$ \\
\hline WSRP-F-01 & $584,285.07$ & $568,346.64$ & $584,908.75$ & $\mathbf{5 5 4 , 4 7 1 . 4 7}$ & $585,321.17$ & $559,036.26$ \\
WSRP-F-02 & $592,505.48$ & $582,181.95$ & $575,441.87$ & $597,279.38$ & $605,906.81$ & $\mathbf{5 5 9 , 1 9 8 . 1 2}$ \\
WSRP-F-03 & $590,040.74$ & $593,763.31$ & $590,619.01$ & $582,329.81$ & $603,655.07$ & $\mathbf{5 8 1 , 5 9 6 . 6 0}$ \\
WSRP-F-04 & $\mathbf{8 2 5 , 9 3 1 . 6 8}$ & $900,387.30$ & $876,679.55$ & $872,606.58$ & $838,692.36$ & $849,852.84$ \\
WSRP-F-05 & $567,245.71$ & $614,704.32$ & $\mathbf{5 4 2 , 3 6 4 . 3 1}$ & $583,121.68$ & $563,245.40$ & $551,663.99$ \\
WSRP-F-06 & $931,935.20$ & $\mathbf{7 1 8 , 3 1 0 . 2 6}$ & $792,308.91$ & $943,102.96$ & $101,4421.53$ & $777,265.11$ \\
WSRP-F-07 & $696,718.60$ & $\mathbf{7 7 7 , 1 6 3 . 3 4}$ & $\mathbf{6 8 4 , 0 8 3 . 3 6}$ & $777,903.33$ & $874,069.14$ & $875,234.10$ \\
\hline Average & $390,006.79$ & $\mathbf{3 8 7 , 0 9 4 . 6 2}$ & $390,031.92$ & $403,632.37$ & $410,449.48$ & $388,798.90$ \\
\hline
\end{tabular}

Bold text refers to the best solution.

problem is presented. The model is constructed by incorporating various constraint formulations from the literature while also adding working region constraints to the formulation. It is usually the case that models in the literature for this type of problem are presented but their solution is approached using alternative methods such as heuristics because solving the model using mathematical exact solvers is computationally challenging. A geographical decomposition with conflict avoidance approach is proposed here to tackle workforce scheduling and routing problems while still harnessing the power of exact solvers. The proposed decomposition method allows to tackle real-world size problems for which finding the overall optimal solution requires too much computation time. However, the solution quality fluctuates when changing the order to tackle the sub-problems defined by the geographical regions. Exploring all permutation orders to find the one producing the best results is not practical for larger problems (e.g. more than 6 geographical areas). In this work, six ordering strategies are proposed for obtaining high-quality solutions within acceptable computation time. Our future research will explore ways to replace the originally defined geographical areas with automated clustering to define well separated geographical areas even in cases where the areas defined by the problem data are not well separated.

\section{ACKNOWLEDGEMENTS}

Special thanks to the Development and Promotion for Science and Technology talents project (DPST, Thailand) who providing partial financial support. Also, we are grateful for access to the University of Nottingham
High Performance Computing Facility.

\section{REFERENCES}

Akjiratikarl, C., Yenradee, P., and Drake, P. R. (2007). PSObased algorithm for home care worker scheduling in the UK. Computers \& Industrial Engineering, 53(4):559583, doi:10.1016/j.cie.2007.06.002.

Angelis, V. D. (1998). Planning home assistance for AIDS patients in the City of Rome, Italy. Interfaces, 28:7583.

Barrera, D., Nubia, V., and Ciro-Alberto, A. (2012). A network-based approach to the multi-activity combined timetabling and crew scheduling problem: Workforce scheduling for public health policy implementation. Computers \& Industrial Engineering, 63(4):802-812, doi:10.1016/j.cie.2012.05.002.

Benders, J. (1962). Partitioning procedures for solving mixed-variables programming problems. Numerische Mathematik, 4(1):238-252, doi:10.1007/BF01386316.

Bertels, S. and Torsten, F. (2006). A hybrid setup for a hybrid scenario: Combining heuristics for the home health care problem. Computers \& Operations Research, 33(10):2866-2890.

Borsani, V., Andrea, M., Giacomo, B., and Francesco, S. (2006). A home care scheduling model for human resources. 2006 International Conference on Service Systems and Service Management, pages 449-454, doi:10.1109/ICSSSM.2006.320504.

Bredstrom, D. and Ronnqvist, M. (2007). A branch and price algorithm for the combined vehicle routing and scheduling problem with synchronization constraints. NHH Dept. of Finance \& Management Science Discussion Paper No. 2007/7, (February).

Bredstrom, D. and Ronnqvist, M. (2008). Combined vehicle routing and scheduling with temporal precedence 
and synchronization constraints. European Journal of Operational Research, 191(1):19 - 31.

Castillo-Salazar, J., Landa-Silva, D., and Qu, R. (2014). Workforce scheduling and routing problems: literature survey and computational study. Annals of Operations Research, doi:10.1007/s10479-014-1687-2.

Castro-Gutierrez, J., Landa-Silva, D., and Moreno, P. J. (2011). Nature of real-world multi-objective vehicle routing with evolutionary algorithms. Systems, Man, and Cybernetics (SMC), 2011 IEEE International Conference on, pages 257-264, doi:10.1109/ICSMC.2011.6083675.

Cordeau, J.-F., Stojkovic, G., Soumis, F., and Desrosiers, J. (2001). Benders decomposition for simultaneous aircraft routing and crew scheduling. Transportation Science, 35(4):375-388, doi:10.1287/trsc.35.4.375.10432.

Dantzig, G. B. and Ramser, J. H. (1959). The truck dispatching problem. Management Science (pre-1986), 6(1).

Dohn, A., Esben, K., and Jens, C. (2009). The manpower allocation problem with time windows and jobteaming constraints: A branch-and-price approach. Computers \& Operations Research, 36(4):1145-1157, doi:10.1016/j.cor.2007.12.011.

Eveborn, P., Ronnqvist, M., Einarsdottir, H., Eklund, M., Liden, K., and Almroth, M. (2009). Operations research improves quality and efficiency in home care. Interfaces, 39(1):18-34, doi:10.1287/inte.1080.0411.

Feillet, D. (2010). A tutorial on column generation and branch-and-price for vehicle routing problems. $4 O R$, 8(4):407-424.

Hart, E., Sim, K., and Urquhart, N. (2014). A real-world employee scheduling and routing application. In Proceedings of the 2014 Conference Companion on Genetic and Evolutionary Computation Companion, GECCO Comp '14, pages 1239-1242, New York, NY, USA. ACM.

Kergosien, Y., Lente, C., and Billaut, J.-C. (2009). Home health care problem, an extended multiple travelling salesman problem. In Proceedings of the 4th multidisciplinary international scheduling conference: Theory and applications (MISTA 2009), Dublin, Ireland, pages 85-92.

Landa-Silva, D., Wang, Y., Donovan, P., Kendall, G., and Way, S. (2011). Hybrid heuristic for multi-carrier transportation plans. In The 9th Metaheuristics International Conference (MIC 2011), pages 221-229.

Liu, R., Xie, X., and Garaix, T. (2014). Hybridization of tabu search with feasible and infeasible local searches for periodic home health care logistics. Omega, 47(0):17 - 32, doi:http://dx.doi.org/10.1016/j.omega.2014.03.003.

Mankowska, D., Meisel, F., and Bierwirth, C. (2014). The home health care routing and scheduling problem with interdependent services. Health Care Management Science, 17(1):15-30, doi:10.1007/s10729-013-92431.

Mercier, A., Cordeau, J.-F., and Soumis, F. (2005). A computational study of Benders decomposition for the in- tegrated aircraft routing and crew scheduling problem. Computers \& Operations Research, 32(6):1451 - 1476, doi:http://dx.doi.org/10.1016/j.cor.2003.11.013.

Perl, J. and Daskin, M. S. (1985). A warehouse location-routing problem. Transportation Research Part B: Methodological, 19(5):381 - 396, doi:http://dx.doi.org/10.1016/0191-2615(85)90052-9.

Pillac, V., Gueret, C., and Medaglia, A. (2012). On the dynamic technician routing and scheduling problem. In Proceedings of the 5th International Workshop on Freight Transportation and Logistics (ODYSSEUS 2012), page id: 194, Mikonos, Greece.

Ralphs, T. K. and Galati, M. V. (2010). Decomposition methods for integer programming. Wiley Encyclopedia of Operations Research and Management Science, doi:10.1002/9780470400531.eorms0233.

Rasmussen, M. S., Justesen, T., Dohn, A., and Larsen, J. (2012). The home care crew scheduling problem: Preference-based visit clustering and temporal dependencies. European Journal of Operational Research, 219(3):598-610, doi:http://dx.doi.org/10.1016/j.ejor.2011.10.048.

Reimann, M., Doerner, K., and Hartl, R. F. (2004). D-Ants: Savings based ants divide and conquer the vehicle routing problem. Computers \& Operations Research, 31(4):563 - 591, doi:http://dx.doi.org/10.1016/S03050548(03)00014-5.

Trautsamwieser, A. and Hirsch, P. (2011). Optimization of daily scheduling for home health care services. Journal of Applied Operational Research, 3:124-136.

Vanderbeck, F. (2000). On Dantzig-Wolfe decomposition in integer programming and ways to perform branching in a branch-and-price algorithm. Operations Research, 48(1): 111 .

Vanderbeck, F. and Wolsey, L. (2010). Reformulation and decomposition of integer programs. In Junger, M. et al., editors, 50 Years of Integer Programming 1958-2008, pages 431-502. Springer Berlin Heidelberg. 